\title{
New insights into America's epidemic of autism spectrum disorders. The simple solution
}

\author{
Strunecka $A^{*}$ and Strunecky $O$ \\ The Institute of Technology and Business, Okružní 517/10, 37001 Ceske Budějovice, Czech Republic
}

Autism spectrum disorders (ASD) cover a range of heterogeneous neurodevelopmental conditions, characterized by persistent deficits in social and communication interactions presenting with repetitive, stereotypic interests, and behaviors [1]. The word autism is traditionally used interchangeably with ASD. While ASD is often considered purely behavioral it comes with many different comorbidities like gut imbalances, seizures, hormonal disorders, obesity, and sleep problems.

ASD is the fastest-growing developmental disability in the last two decades in all countries over the world. ASD prevalence in the USA was the highest in $2016^{\text {th }}$ data within all developed countries with an average value of 250 per 10,000 (one in 40) children aged 3-17 years [2]. It further increased since the previous survey in 2014 when one in 59 among eight-year-old children was diagnosed with ASD in the US [3].

The ASD etiopathology involves numerous mechanisms, but most ASD risk factors are connected either directly or indirectly to immunoexcitotoxic processes [4]. Inflammation of the brain has been reported by several authors in postmortem brain specimens of both young and old individuals with ASD [4]. The initial reaction to systemic inflammation might either impact microglial priming or full neurotoxic activation. Great number of conditions leads to the priming/activation of microglia, such as stress, obesity, trauma, infections, hypoxia-ischemia, as wells as exposure to a number of environmental excitotoxins and certain neurotoxic metals, such as mercury, lead, and aluminum. We demonstrated that fluoride could exacerbate pathological problems by worsening excitotoxicity and inflammation [5].

A long-term burden of fluoride has several health effects with a striking resemblance to the symptoms of ASD, such as inflammation, impaired the ability of cognition and IQ deficits, sleep-pattern disturbance, learning and behavioral problems in some individuals [5]. Prolonged exposure to fluoride in the prenatal and early postnatal stages might have toxic effects on the development and metabolism of the brain. Moreover, fluoride interferes with various enzymes, resulting in significant suppression of cellular energy production and oxidative stress [6]. Despite intensive research, effective prevention of ASD symptoms supported by evidence-based medicine has not been found.

Fluoride is a ubiquitous compound found in drinking water (both natural and supplemented), in tea, marine fish, and in fluoridated toothpaste. In 2014 , the CDC reported that $75 \%$ of the US population has been supplied with fluoridated water for 70 years. Our suggestion on the significant role of fluoride in the ASD etiopathogenesis is supported by the observation that a high ASD rate is found in countries with a high occurrence of dental fluorosis. Dental fluorosis is an undesirable side effect of excessive fluoride intake during the childhood. The last US survey found dental fluorosis in 65\% of adolescents aged 12-15 years [7]. The US Environmental Protection Agency (EPA) has done both a dose-response analysis and a relative source contribution analysis [8] that showed that a third of children between the ages of 6 months and 4 years were getting significantly more fluoride than is considered safe. For infants up to 6 months old receiving infant formula using drinking water with fluoride level higher than $0.8 \mathrm{mg} \mathrm{L}^{-1}$ the intake of fluoride will exceed $0.1 \mathrm{mg} \mathrm{kg}^{-1} \mathrm{day}^{-1}$. This fluoride concentration is 100 times higher than the concentration found in breast milk (less than $0.001 \mathrm{mg}$ $\mathrm{kg}^{-1}$.

The toxic action of fluoride has been attributed to its function as an enzymatic poison, inhibiting activities of many important enzymes [6]. Fluoride is responsible for numerous metabolic disorders and results in a decrease in energy metabolism, redox potential of the cells, and an increase in oxidative stress.

Almost 50\% of children with ASD display peripheral markers of disturbances in mitochondrial energy metabolism [9]. Young children, particularly between age of 4 and 30 months with dysfunctional cellular energy metabolism might be inclined to autistic regression especially in case of their genetic susceptibility, infections or immunizations at the same time.

Recently, Delhey et al. measured mitochondrial enzyme activity on one of the largest cohorts of individuals with ASD studied to date with concurrent measurement of symptoms in a subset and found that children with ASD demonstrated significantly greater variation in mitochondrial activity compared to controls [10]. This study demonstrates, for the first time, that such metabolic variations are related to ASD symptoms.

We found that 315 laboratory, clinical, epidemiological, and ecological studies over the whole world brought evidence about fluoride neurotoxicity [5]. The effect of chronic fluoride exposure on children's intelligence measured as intelligence quotient (IQ) has been traditionally investigated as an indication of the neurotoxic effect of fluoride in various geographical areas. The strength of association between higher fluoride concentrations in the water and children's reduced intelligence was further supported by a dose-response metaanalysis [11]. These authors evaluated 26 studies of 7258 children and suggested that exposure to fluoride should be controlled in areas

*Correspondence to: Anna Strunecka, The Institute of Technology and Business, Okružní 517/10, 37001 Česke Budějovice, Czech Republic, E-mail: anna. strunecka@gmail.com

Received: December 13, 2019; Accepted: December 20, 2019; Published: December 23, 2019 
with high fluoride levels in the water. Almost half (44\%) of children identified with ASD in the US have average to above average intellectual ability [12].

In the recent Canadian study, maternal exposure to higher levels of fluoride during pregnancy was associated with lower IQ scores in children aged 3 to 4 years [13]. A $1 \mathrm{mg}$ higher daily intake of fluoride among pregnant women was associated with a 4.49-point lower IQ score in boys, but there was no statistically significant association with IQ scores in girls. These findings support the possible need to reduce fluoride intake during pregnancy. The prevalence rates of ASD are also on the rise in Canada. While in 2003, one in 204 children had a diagnosis of autism/ASD, this rate rose to approximately one in 66 in 2018 [14]. As of 2007, 45.1\% of the Canadian population had access to fluoridated water supplies and water fluoridation remains a contentious issue. The Canadian Health Measures Survey found that $16 \%$ of children may have very mild or mild dental fluorosis.

Based on the ASD prevalence in various countries and cities, we suggest that fluoride might be the significant culprit in the ASD etiopathogenesis both in areas with artificial water fluoridation as well as in fluoride endemic areas [5]. We showed that diverse biological processes were affected by fluoride including neurotransmission and hormonal regulation. Under such circumstances, a "safe" fluoride concentration might induce pathological effects in children with genetic susceptibility. The heterogeneity of mutual dynamic interactions can explain the clinically heterogeneous symptoms of ASD. The rise of ASD in the last decades challenges us to change the research of the ASD etiopathogenesis from a reductionistic approach to an understanding its underlying integrative networks.

ASD may significantly limit the capacity of some individuals to conduct daily activities. Since it is a life-long condition it often imposes a significant socioeconomic burden on ill people, their families, and on whole society. In the US, the annual societal costs per the year 2011 for children with ASD were estimated to be between \$11.5-60.9 billion, including a variety of direct and indirect costs, from medical care to special education and lost parental productivity. Children and adolescents with ASD had average medical expenditures that exceeded expenditures of those without ASD by $\$ 4110-6200$ per year [12]. According to estimates, annual economic losses due to ASD in the US in 2015 were around $\$ 268$ billion. This figure is estimated to increase to about $\$ 461$ billion by the year 2025 [15]. The economic impact of IQ loss among US children yearly costs tens billions of dollars.

Fluoride has no known essential function in human growth and development and no signs of fluoride deficiency have been identified [16]. At present, there is a divergence between the public health practice of water fluoridation, which is regarded as valuable and safe for reducing dental caries, and current scientific evidence, which indicates that fluoride is a neurotoxin disturbing prenatal as well as postnatal brain development, eroding intelligence, and behavior. The ASD prevalence is consistently estimated as a ratio of approximately 4.5 male: 1 female during 2006-2014 in the US [12]. The perspective that ASD could impact mostly young men in the next decades is very alarming and requires urgent solutions.

We suggest that the reduction of fluoride exposure of pregnant women and developing children in daily life could be a very easy and inexpensive way to prevent an ASD epidemic. Monitoring of the ASD prevalence in children born after the removal of fluoride from drinking water will provide relevant information for our hypothesis.

\section{Acknowledgement}

A. Strunecka was retired from the Faculty of Sciences, Department of Physiology and Developmental Biology and the 1st Faculty of Medicine, Laboratory of Biochemical Neuropharmacology, Charles University in Prague.

\section{Conflicts of interest}

The authors declare no conflict of interest.

\section{References}

1. American psychiatric association. diagnostic and statistical manual of mental disorders (DSM-5®) (2013) American Psychiatric Pub 22: 991.

2. Kogan MD, Vladutiu CJ, Schieve LA, Ghandour RM, Blumberg SJ, et al. (2018) The prevalence of parent-reported autism spectrum disorder among US children. Pediatrics 142: e20174161.

3. Baio J, Wiggins L, Christensen DL, Maenner MJ, Daniels J, et al. (2018) Prevalence of autism spectrum disorder among children aged 8 years-Autism and developmental disabilities monitoring network, 11 Sites, United States, 2014. MMWR Surveill Summ 67: $1-23$

4. Strunecka A, Blaylock RL, Patocka J, Strunecky O (2018) Immunoexcitotoxicity as the central mechanism of etiopathology and treatment of autism spectrum disorders: A possible role of fluoride and aluminum. Surg Neurol Int 9: 74-74.

5. Strunecka A, Strunecky O (2019) Chronic fluoride exposure and the risk of autism spectrum disorder. Int J Environ Res Public Health $16: 3431$.

6. Strunecka A, Patocka J, Blaylock RL, Chinoy NJ (2007) Fluoride interactions: From molecules to disease. Curr Signal Transduct Ther 2: 190-213.

7. Neurath C, Limeback H, Osmunson B, Connett M, Kanter V, et al. (2019) Dental fluorosis trends in US oral health surveys: 1986 to 2012. JDR Clin Trans Res 4: 298308 .

8. EPA (2011) Fluoride: Exposure and relative source contribution analysis documents.

9. Rose S, Niyazov DM, Rossignol DA, Goldenthal M, Kahler SG, et al. (2018) Clinical and molecular characteristics of mitochondrial dysfunction in Autism spectrum disorder. Mol Diagn Ther 22: 571-593.

10. Delhey L, Kilinc EN, Yin L, Slattery J, Tippett M, et al. (2017) Bioenergetic variation is related to autism symptomatology. Metab Brain Dis 32: 2021-2031.

11. Duan Q, Jiao J, Chen X, Wang X (2018) Association between water fluoride and the level of children's intelligence: A dose-response meta-analysis. Public Health 154: 87-97.

12. CDC (2018) Data \& Statistics on Autism Spectrum Disorder. (2018) Centers for Disease Control and Prevention.

13. Green R, Lanphear B, Hornung R, Flora D, Martinez-Mier EA, et al. (2019) Association between maternal fluoride exposure during pregnancy and IQ scores in offspring in Canada. JAMA Pediatr. Aug 19.

14. Autism Spectrum Disorder among children and youth in Canada (2018) Available online: https://www.canada.ca/en/public-health/services/publications/diseases-conditions/autismspectrum-disorder-children-youth-canada-2018.html (Accessed on 1 June 2019).

15. Leigh JP, Du J (2015) Brief report: Forecasting the economic burden of autism in 2015 and 2025 in the United States. (2015) J Autism Dev Disord 45: 4135-4139.

16. EFSA (2017) Dietary Reference Values for nutrients. Summary report (2017) EFSA Supporting publications $14: 29-30$.

Copyright: (C2019 Strunecka A. This is an open-access article distributed under the terms of the Creative Commons Attribution License, which permits unrestricted use, distribution, and reproduction in any medium, provided the original author and source are credited. 